https://doi.org/10.5719/aub-g/68.1/2

\title{
PROBLÉMATIQUE DE LA MOBILITÉ URBAINE DANS LES VILLES ALGÉRIENNES. CAS DE LA CONGESTION ROUTIÈRE À BEJAIA
}

\author{
IDJERAOUI OUAHIBA ${ }^{1}$, BOUTABBA HYNDA ${ }^{2}$, MILI MOHAMED ${ }^{3}$
}

\begin{abstract}
Because of its importance and its impact, urban mobility in major Algerian cities is a topical issue of great importance, given the critical state of congestion caused by transport congestion, particularly in urban centers. Due to its large population, its strategic position at the crossroads of four national highways and its large-scale socio-economic facilities, the city of Bejaia (the second largest city in Algeria for port business activities) has an attractive power over the entire region, resulting in significant commuting, with a clear preference for private cars and effective use of public transport. However, its road and transport networks suffer from several hazards, which has had negative effects on road traffic. To remedy this situation, the local authorities have taken several actions to correct the malfunctions of the traffic. This paper attempts to highlight the role of local governance in managing urban mobility. Our main question concerns the current actions implemented by local authorities in order to curb the congestion that characterizes this city.
\end{abstract}

Keywords: local governance, urban mobility, congestion, urban transport, Bejaia.

Résumé

De par son importance et ses impacts, la mobilité urbaine dans les grandes villes algériennes, constitue un sujet d'actualité d'une grande importance, vu l'état critique dû à la congestion des transports qu'elles connaissent, notamment dans les centres-villes.

En raison de sa forte population, sa position stratégique au carrefour de quatre routes nationales et ses équipements socio-économiques de grande envergure, la ville de Bejaia (Deuxième ville d'Algérie, en matière d'activités commerciales portuaires) exerce un pouvoir attractif sur l'ensemble de sa région, entraînant des mouvements pendulaires importants, avec une nette préférence de la voiture particulière et une utilisation effective des transports en commun. Cependant, ses réseaux viaires et de transport souffrent de plusieurs aléas, ce qui avait engendré des effets négatifs sur la circulation routière. Pour remédier à cette situation, les autorités locales ont entrepris plusieurs actions, afin de corriger les dysfonctionnements de la circulation.

1 Doctorante Institut de Gestion des Techniques Urbaines. Université de Constantine 3. Ouahiba.Idjraoui@univ-constantine3

2 Professeur, Institut de Gestion des Techniques Urbaines. Université de M'sila. hynda.boutabba@univ-msila.dz

3 Maître de conférence, Institut de Gestion des Techniques Urbaines. Université de M'sila. Mohamed.mili@univ-msila.dz 
Le présent papier essaye de mettre en exergue le rôle de la gouvernance locale en ce qui concerne la gestion de la mobilité urbaine. Notre principale interrogation porte sur les actions actuelles mises en cuvre par les collectivités locales afin d'endiguer la congestion qui caractérise cette ville.

Mots-clés : gouvernance locale, mobilité urbaine, congestion, transport urbain, Bejaia, Algérie.

\section{Introduction}

Depuis son indépendance en 1963, notamment à partir du moment de l'approbation du code d'investissement de 1966, l'État Algérien avait opté pour une stratégie d'industrialisation poussée des secteurs de la sidérurgie et de la pétrochimie, placés tout au Nord du pays (Mira, 2015), ce qui avait encouragé les ruraux déracinés des steppes, des plaines et des montagnes qui se sont agglutinés massivement autour des grandes villes (Cote, 1979; 1983 ; Mutin, 1980 ; Boutabba, 2013). Pour répondre à la demande croissante de mobilité des personnes, de transport et de fourniture de marchandise, la construction routière fut une des priorités de la politique de développement du territoire de l'Algérie (Leveque et Fidéli, 2017, p.1). Cependant depuis les années 1990, date à laquelle l'Algérie s'était engagée dans un processus de transition d'un système économique centralisé à une économie de marché, suite à une série de réformes structurelles qui avaient abouti à la libéralisation de l'économie (BennacerKhaladi, 2011), le pays a connu une demande de plus en plus croissante et exigeante en matière de mobilité (Belouachrani, 2009; Berchache, 2011). Phénomène de société lié notamment à l'urbanisation (Baouni et Hadjiedj, 2000) et à l'activité économique, qui se manifeste par un accroissement très important de la circulation et du parc automobile (Auburtin et al, 1996, P. 13). Pour faire face à cette demande, un grand nombre de projets ont été récemment programmés dans le pays, certains sont déjà réalisés d'autres sont en phase de réalisation (Azzag, 2000; Chennaoui et al., 2000, Messaoudène et Boutabba, 2017), alors que d'autres sont actuellement gelés à cause de l'austérité (Merzoug et Belkhiri, 2010) et la crise financière que connait le pays.

A l'instar des autres villes algériennes, Bejaia n'échappe pas à ce phénomène, en effet, la circulation est de plus en plus croissante, et la ville est confrontée à des congestions de plus en plus ressenties par les usagers de la route (Benkhnnouche, 2012 ; Baouni et al., 2013 ; Sali, 2015 ; Idjraoui et al., 2016). Ce problème est lié à plusieurs facteurs : sa situation géographique stratégique, favorisant les mouvements pendulaires et transitaires ; la concertation d'équipements administratifs et culturels d'une considérable ampleur ; des infrastructures économiques de grande envergure tels que son port pétrolier et son aéroport international (Kheladi, 1998, Sadoudi, 2013) ainsi qu'une concentration humaine assez importante (Fig. 1). Rappelons que la population de la commune est estimée à 188250 habitants (DPSB, 2016) avec 
un parc automobile important et un taux de motorisation de 22057 véhicules (SCG, 2016). Malgré ces nombreux atouts, le réseau routier de la ville de Bejaia, est actuellement en inadéquation par rapport aux exigences du trafic routier grandiloquent (Kheladi, 2002 ; Merzoug, 2018). Ceci revient à différents aléas en matière des infrastructures de transport, et notamment d'infrastructures routières. En effet, Le système viaire souffre de saturation, de dégradation et de stationnement anarchique.

Partant du postulat que la mobilité et la fluidité des déplacements sont parmi les facteurs essentiels qui assurent une bonne gestion de la ville, il est impératif que les localités territoriales assoient une politique claire en matière d'exploitation et d'entretien de transport et du réseau routier (De Gayffier, 2004 ; Belouachrani, 2009). A travers cette interventions nous allons essayez de mettre sous la loupe les actions actuelles mises en œuvre par les collectivités locales afin d'endiguer la congestion qui caractérise la ville de Bejaia. L'objectif étant de maitre en avant la situation critique de mobilité a Bejaia et de mettre en exergue les politiques de gestion locales adoptées pour améliorer la situation
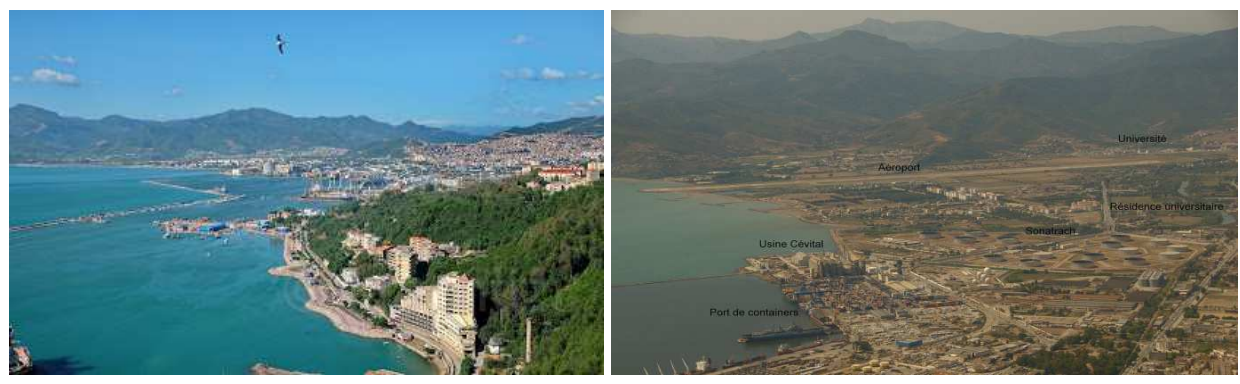

Fig. 1. Bejaia une ville très convoitée : des infrastructures économiques d'envergure (Source : les auteurs)

\section{Méthodologie}

Dans l'optique d'apporter des réponses aux questionnements posés, de vérifier l'action effective des collectivités locales et de détecter les contraintes rencontrées dans la gestion urbaine des transports dans la ville de Bejaia, un travail de terrain, en deux étapes essentielles, a été indispensable.

Afin de ressortir les défaillances qui ont un impact direct sur la mobilité urbaine dans la ville de Bejaia, la première étape s'est attelée à dresser un état de lieux du réseau routier et des différents modes de transport urbain opérationnels. Cette étape a fait appel à la technique de l'observation directe (Simiand, 1983 ; Bogdan et Taylor1975) ainsi qu'à la collecte des statistiques auprès des gestionnaires du réseau routier et du réseau de transport. 
La deuxième a porté sur l'identification du processus de gestion locale de la mobilité urbaine par les services publics. L'analyse des politiques de gestion d'exploitation et d'entretien du réseau routier, ainsi que celle liée au contrôle et à la prévention du trafic routier ont été exécutées en maniant la technique de l'entretien semi directif, qui «favorise un déplacement du questionnement, tourné vers le savoir et les questions propres des acteurs sociaux» (Michelat et Michel Simon, 1977 ; Duchesne, 2000). L'enquête s'est déroulée sur une période de 6 mois, allant du 15 octobre au 25 Avril 2016 et a ciblé un échantillon représentant les différents gestionnaires de la ville étatiques et privés. Un total de 25 entretiens a été exécuté. Ce corpus nécessaire à la réalisation de l'enquête s'est avéré suffisant, dans la mesure où les informations issues des entretiens sont validées par le contexte et n'ont pas besoin de l'être par leurs probabilités d'occurrence (Becker, 2002 ; Blanchet et Gotman, 2007).

\section{Présentation de la zone de l'étude : l'état des lieux du réseau routier}

Le département de Bejaïa est situé au Nord-est de l'Algérie. Ile est situé à $250 \mathrm{Km}$ à l'est de la capitale algérienne sur le littoral algérien. D'une superficie de 322348 hectares, il est divisé administrativement en 52 communes et 19 daïras. Sa population avoisine selon le recensement de 2014, 185882 habitants. Ce département se singularise par l'occupation d'un site qui est une synthèse des principaux reliefs (mer, montagne, plaine, vallée, rivière et piémont) (PDAU, 1997), influençant ainsi l'état et l'exploitation du réseau de la voirie. Ce département est desservi par un réseau routier assez étoffé, constitué de plusieurs routes nationales : la RN 12 reliant Bejaia à la capitale, la RN 26 reliant El Kseur-Bouira, la RN 09 la reliant à Sétif et à Djidjel, la RN 24 reliant Béjaia - Tizi Ouzou et les RN 74 et 75 reliant Béjaia - Bordj Bou Arréridj (Fig. 2).

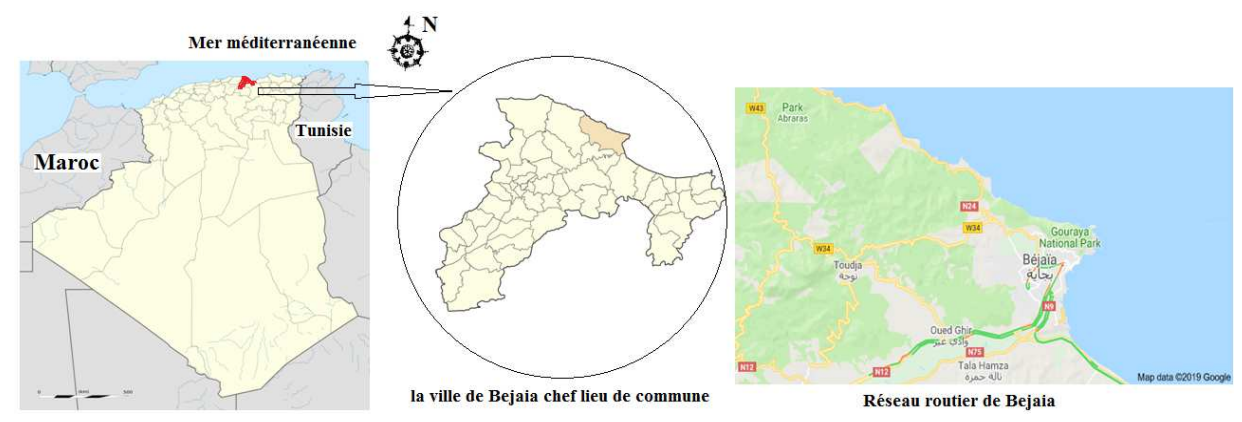

Fig. 2. Situation géographique et réseau routier de Bejaia 
Situé au cœur de l'espace méditerranéen, le chef-lieu de commune (la capitale administrative du département) : la ville de Bejaia, s'étend sur une superficie de $120,22 \mathrm{Km}^{2}$ et compte 188250 habitants, pour une densité de $1546,18 \mathrm{ha} / \mathrm{Km}^{2}$ (DPSB, 2016). Par sa situation géographique, la ville de Bejaia est considérée comme l'une des plus stratégiques que compte le département et tout le nord algérien, en étant l'une des villes de $1^{\text {er }}$ ordre occupant le centre de la bande littorale de l'Afrique du Nord. Ainsi son avantageuse localisation géographique a fait d'elle un important pôle d'attraction (PDAU intercommunal, 2009) et un lieu très fréquenté. D'un autre coté, sous l'effet d'une croissance naturelle et d'un exode rural massif, la population de la ville de Bejaia a augmenté de $56 \%$ de 1987 à 2013. Parallèlement, suite à la libéralisation du marché foncier et immobilier durant les années 1990, le périmètre urbain de la commune de Bejaia a doublé entre 1987 et 2009 (Tableau.1). À cette concentration de la population dont la mobilité est amplifiée par l'étalement urbain, s'ajoutent des flux en provenance des communes limitrophes (Merzoug, 2017), d'où la nécessité d'un système circulatoire fonctionnel et organisé. Malheureusement, ceci ne semble pas être le cas actuellement, vu les nombreux problèmes de congestion observés dans la ville. Ces congestions constituent un véritable frein à l'exploitation de diverses potentialités que peut offrir cette ville et donc une entrave à son développement.

Evolution des surfaces urbanisées de Bejaia

Tableau 1

\begin{tabular}{|c|c|c|c|}
\hline Année & 1987 & 1997 & 2009 \\
\hline Surface (Ha) & 1300 & 1760 & 2917,92 \\
\hline
\end{tabular}

Source : PDAU intercommunal, 2009

Selon Colletis-Wahl \& Meunier (2003), le réseau routier est définit en science de l'aménagement du territoire, comme étant un ensemble de mailles sur un territoire donné. Il est composé de voies de communications reliant des agglomérations humaines, soit un ensemble d'axes routiers desservant différents commune, villes, départements et région. La gestion de la mobilité urbaine est généralement la résultante d'interaction entre le réseau routier, le réseau de transport et les autres composantes de déplacement urbain à savoir : les modes doux et les véhicule tous types confondus (trafic routier et la dépendance à la voiture particulière). Ainsi, une bonne connaissance de la situation actuelle permet une bonne compréhension de la problématique.

Le réseau de desserte de la ville de Bejaia est organisé d'une façon hiérarchique. Il comprend un réseau principal, entourant le périmètre urbain, assurant la liaison entre les différentes zones de la ville. Il s'agit des boulevards Krim Belkacem, Route de Aurès, Boudchicha Tahar situés au centre-ville. Boulevard de la Soummam reliant la zone Est de la ville. Moulay En Nacer, 
Mustapha Ben Boulaid, desservant le port. Boulevard de la liberté considéré comme axe de centralité administrative. Les voies secondaires inter quartiers, parmi lesquelles, nous citons dans une liste non exhaustive, les rues Tabet, Boumdaoui permettant l'accès à la zone industrielle et la rue frères Tifaoui permettant la desserte de l'arrière port. Quant au réseau tertiaire, il assure les dessertes à l'intérieur des quartiers. Au centre-ville, ce réseau est assez bien maillé et relativement dense. En périphérie tels que relatif à la vieille ville, Ihidadene, Ighil, Sidi Ahmed, il est plutôt tortueux et relativement accidenté (Fig. 3 et 4 ).

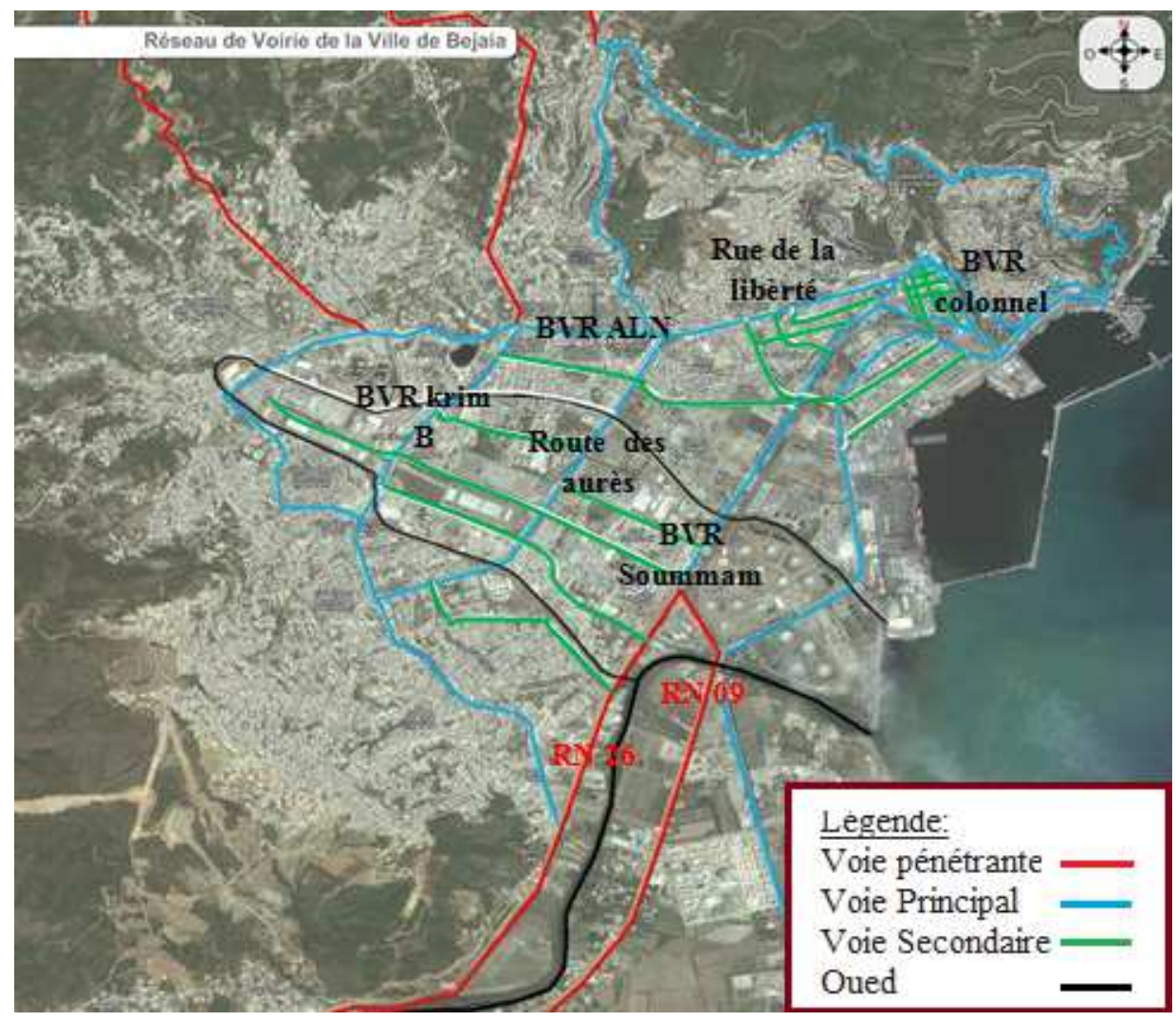

Fig. 3. Les axes routiers de la ville de Béjaia (Source : Plan de transport et de circulation de la ville de Bejaia, 2014) 

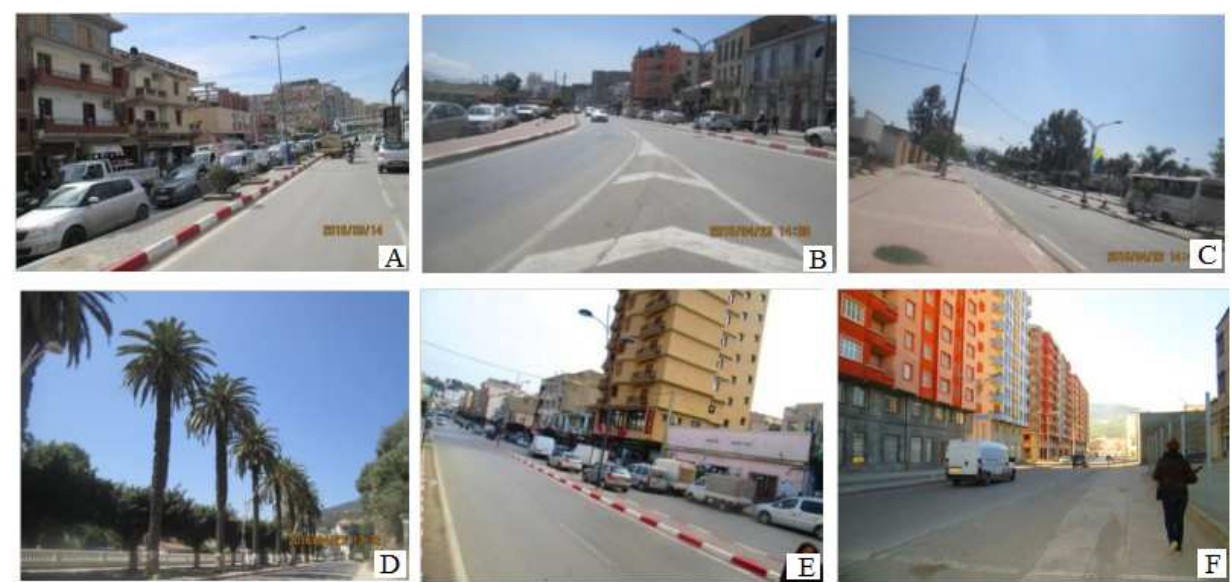

Fig. 4. Grandes artères de la ville de Bejaia

A : Avenue Touati, B : Boulevard de la Soummam, C Boulevard Moulay En Nacer,

D : Boulevard Mustapha Ben Boulaid, E : Rue de la liberté, F : Rue Boumedaoui

(Source : les auteurs)

En somme, plus de $300 \mathrm{Km}$ du réseau de voirie urbaine sont concentrés au niveau de la commune de Bejaia, dont $20 \%$ sont en mauvais état et $20 \%$ en moyen état (DTP, 2015). Les réseaux principaux et secondaires présentent de bonnes caractéristiques géométriques, cependant ils diffèrent par rapport à leur état d'entretien. Alors que le premier est généralement en bon état, le second est mal entretenu. Quant au réseau tertiaire, il représente de mauvaises caractéristiques géométriques notamment des ruelles étroites et majoritairement en mauvais état à l'exception faite de celui du centre-ville (Fig. 5).

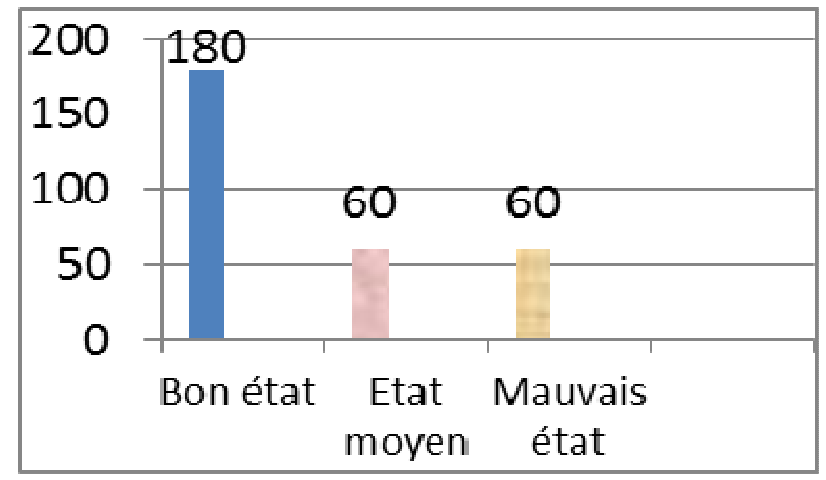

Fig. 5. Etat du réseau routier à Bejaia

(Source: Auteurs selon les statistiques de la direction des travaux publique 2015) 
Comme il est à remarquer aussi une absence totale des pistes pour les modes doux (pistes cyclables, piétonnes), un manque et détérioration en matière de signalisation routière à plusieurs endroits, ainsi qu'une insuffisance en matière de stationnement conduisant à des stationnements anarchiques sur voirie. De même, l'éclairage public le long des axes routiers souffre d'après les services de l'APC (2015) d'une défaillance technique et ne fonctionne pas sur une importante distance évaluée à $762.4 \mathrm{~m}$. Cette situation affecte amplement le niveau de service ce qui n'est pas sans conséquence sur la fluidité du trafic routier et donc participe aux problèmes de congestion que connait la ville. Cet état de fait nous a incités à nous interroger sur les politiques de gestion d'exploitation et d'entretien du réseau routier?

\section{Différents modes de transport terrestre opérationnels à Bejaia}

Dans la ville de Bejaia, les modes de déplacement motorisés sont assurés par deux principaux modes de transport terrestre : le transport en commun (bus et taxis de service) et les véhicules personnels. Malheureusement les réseaux empruntés souffrent de plusieurs aléas.

\subsection{Défaillances techniques du réseau de transport urbain routier}

En adoptant des plans de transport, les autorités publiques gestionnaires des villes de par le monde, espèrent généralement assurer les besoins en mobilité de tous leurs habitants. Le but étant de réduire de manière efficace et efficiente la dépendance à l'automobile et en favorisant l'essor du transport collectif et actif, et ce, au bénéfice d'une ville plus verte et plus saine (Beaudet et Wolff, 2003). En Algérie, cette idée commence à germer depuis l'année 1987, date de la libéralisation du transport urbain. Depuis, plusieurs opérateurs privés ont vu le jour suite aux innombrables encouragements économiques faits par l'état. En effet, nombreux sont les jeunes chômeurs qui avaient bénéficié de crédits bancaires bonifiés et des exonérations fiscales assez alléchantes. L'Etat algérien considérait le créneau du transport public urbain comme un moyen d'excellence pour lutter contre le chômage qui était très élevé, notamment après la crise pétrolière de 1986 et la décennie politique noir des années 1990. Ainsi dans toutes les grandes villes algériennes prolifèrent plusieurs sociétés privées de transport collectif public, notamment type minibus, Fourgons et Autocars. Cependant, sous le double effet de la forte croissance urbaine et humaine de ces villes ainsi que l'absence d'une réglementation

\footnotetext{
$4 \quad$ Assemblée populaire communale (commune).
} 
définissant les règles minimales d'exploitation et de concurrence du secteur privé de transport public, l'offre se trouve vite dépassée par la forte demande des habitants. Cet état de fait avait contribué, notamment depuis cette dernière décade des années 2010, à la reconquête de l'exploitation du transport urbain par le secteur public, et ce via, l'intégration d'autobus robuste d'une capacité d'une centaine de 100 places à travers tout le territoire national. A Bejaia, cette opération a été faite grâce à l'ÉTUSB qui a pu désengorger le secteur de transport à raison de $17,2 \%$ du total des exploitations.

Bejaia est desservie par un réseau de transport en commun (TC) important couvrant un linéaire d'environ $180 \mathrm{~km}$ avec une fréquentation annuelle d'enivrons 25 millions de voyages. La ville dispose d'un réseau de transport urbain et d'une bonne couverture spatiale de ce dernier, sauf au niveau de la zone industrielle. Ce réseau est constitué de 29 lignes de TC, actuellement opérationnelles assurées par les opérateurs privés, qui sont en nombre de 308 ainsi qu'un parc de 356 bus et 5 lignes urbaines assurées par le seul opérateur public E.T.U.S.B, disposant un parc de 29 véhicules, mis en circulation quotidiennement sur son espace. Il est également constitué de 22 lignes de transport universitaire avec un parc de 86 bus universitaires $\left(\mathrm{DTW}^{5}, 2017\right)$ ainsi que 20 stations de taxis $(13$ stations urbaines et 7 inters-urbaines) réparties sur son territoire (Fig. 6).

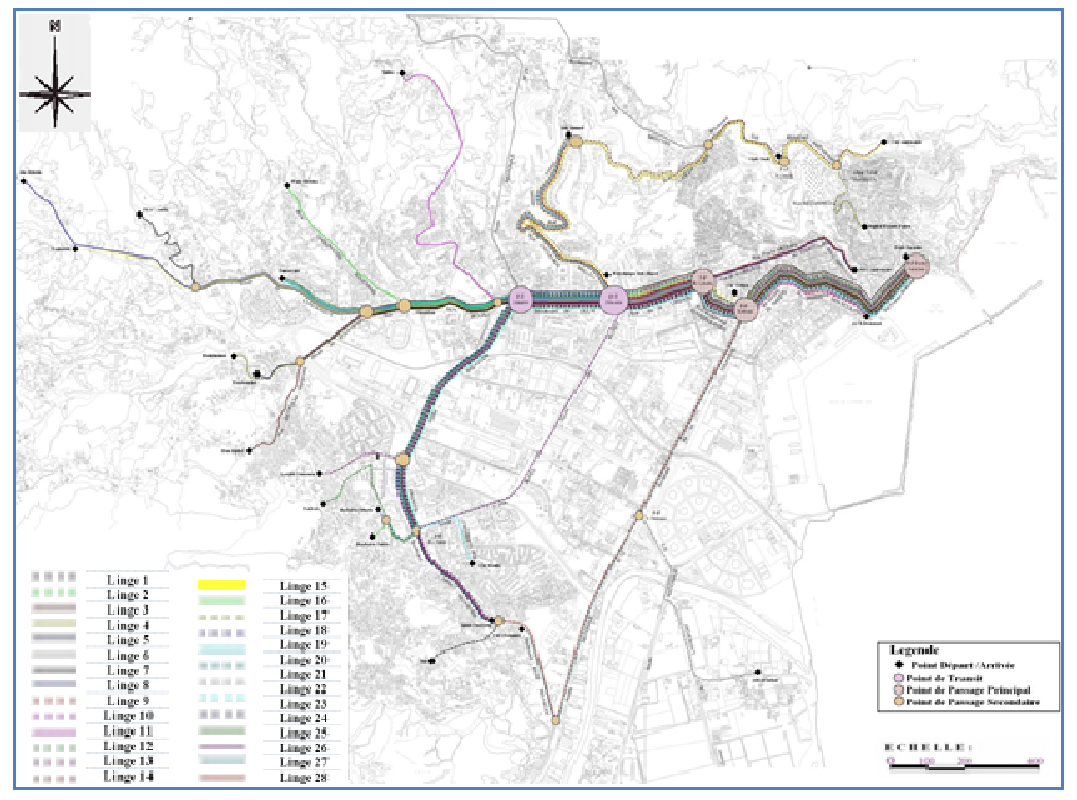

Fig. 6. Ligne de transport urbain privé de la ville de Bejaïa (Source : direction des transports de la wilaya de Bejaia)

5 Direction de transport de la wilaya 
Si on se réfere seulement à ces statistiques l'offre de transport parait admissible, mais, en réalité, plusieurs problèmes sont recensés. Parmi ceux-ci nous citons :

- Absence d'espace réservé au transport collectif urbain ou sites propres. En effet, les véhicules dédiés au transport collectif à Bejaia circulent au milieu des automobiles, ce qui accentue l'encombrement, diminuent leurs rendements et les rend peu attrayants aux yeux des utilisateurs. Ces derniers les assimilent à des malaises urbains que des facilitateurs de vie urbaine (Lebreton et Beaucire, 2000).

- L'arrêt simultané de plusieurs bus en même temps gêne énormément la circulation. Ceci est d'autant plus accentué par le manque d'aménagement réservé à l'arrêt de ces bus et parfois même absence de plaques signalant leurs existences. Si l'emploi des abribus comme mobilier urbain s'est généralisé dans toutes les grandes agglomérations françaises depuis 1964, et par la suite dans la majorité des villes de l'Europe du Nord (Gröndahl, 2016) ; à Bejaia, et à l'exception faite de certains arrêts du centreville, la majorité des arrêts voués aux véhicules du transport urbain sont dépourvus d'abribus ; et s'ils existent, leurs chaises sont abimées, parois cassés ou plein de graffitis. On est loin de l'idée du contrat social devant être passé entre des responsables politiques qui acceptent que ces équipements privés soient distribués dans les rues en vue d'améliorer l'usage public des transports urbains et des sociétés privées qui les exploitent (Pégard, 2007). Cette absence de matérialisation claire des arrêts de bus a conduit à la pratique de "l'arrêt sur commande » qui s'exécute suite à la demande d'un utilisateur même dans des endroits non propices à l'arrêt, mettant parfois la sécurité de cet utilisateur en danger, car aujourd'hui, la sécurité routière se juge davantage à l'aune du faible risque pour l'usager qu'à celle du faible nombre d'accidents (Davis, 1992). Cette pratique est généralement remarquée pour les opérateurs privés qui couvrent plus de 13.482 places soit $82,8 \%$ du total des prestations de ce créneau.

- Concentration des transports en commun sur les principales voies de la couronne interne de la ville au niveau de la plaine telle que la rue de la liberté, le boulevard ALN et le boulevard Krim Belkacem (DTW, 2017) ce qui provoque de longues files de circulation (Fig. 7), créant ainsi un grand encombrement, contraignant de plus en plus la fluidité du trafic (BETT, 2015). La desserte spatiale des rues tortueuses et sinueuses de la vieille ville vient en deuxième position, ce qui crée un déséquilibre du système de transport. Pour le reste de la ville, la répartition en matière de transport en commun est faite selon la taille des véhicules utilisés par les opérateurs. En raison de l'utilisation des minicars de 35 places, le secteur privé assure la liaison entre les quartiers périphériques et le centre-ville, comme il assure, à un degré minime, la desserte des 
quartiers de la haute ville. A l'inverse, vu l'utilisation de grands véhicules de 100 places et plus, l'opérateur ÉTUSB dessert plutôt les quartiers principaux à partir des accès principaux à la ville, via les routes nationales 9 et 12 .

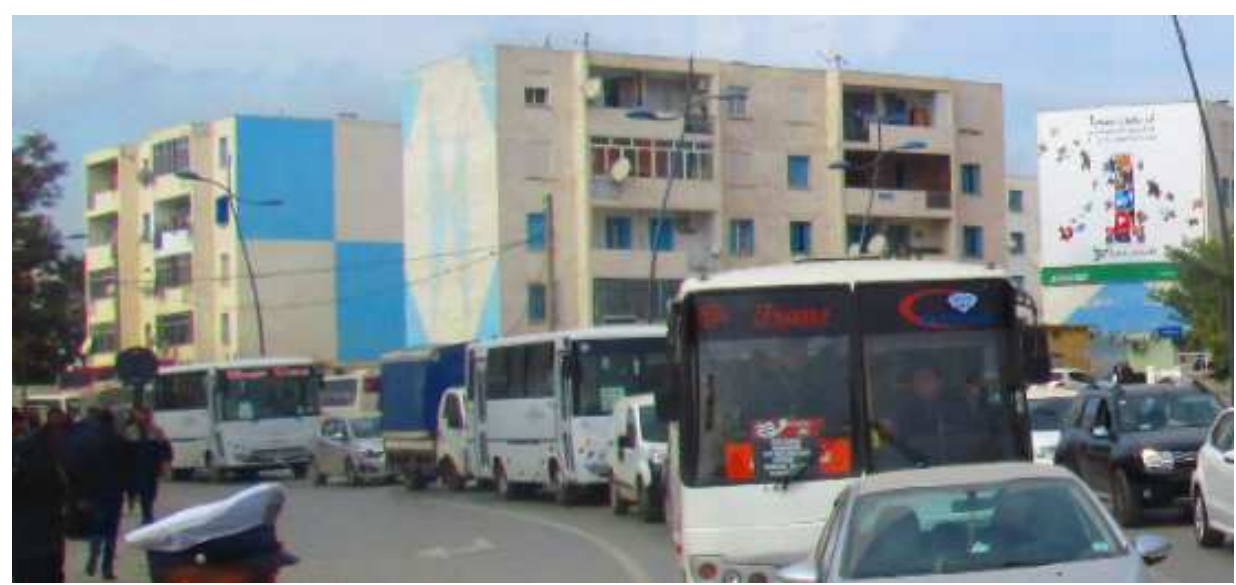

Fig. 7. Concentration du transport en commun au niveau la rue de la liberté (Source : le auteurs, 2017)

- Le vieillissement du parc du transport public urbain est aussi à prendre en considération. En Algérie le parc autobus est considéré comme relativement vieux, notamment celui des opérateurs privés. Les véhicules utilitaires légers tous carburants confondus de 4 ans d'âge atteignent un kilométrage annuel moyen de $31.389 \mathrm{~km}$ (Boughédaoui, 2009). A Bejaia, 94,30\% du parc du transport public urbain à plus de 5 ans (CTW, 2017). Ces différents aléas ont un impact négatif sur les performances du secteur transport et sur la fluidité de la circulation dans cette ville.

- L'absence d'aménagement des trois stations urbaines qui représentent les principales gares d'attache pour un certain nombre de lignes des différentes destinations : stations porte Sarazine, station Sid Ahmed et station Boulevard Amirouche ;

Quant à l'utilisation de la voiture particulière par la population Bougeotte, il est à remarquer qu' actuellement dans la ville de Bejaia, la voiture particulière est en tête de liste dans le choix du mode de déplacement. L'étalement urbain que connait cette ville depuis cette dernière décade des années 2010 a favorisé le recours aux voitures particulières qui tendent à converger simultanément vers le centre des agglomérations et alimentent la congestion urbaine. Ce phénomène n'est pas propre à Bejaia, mais national ou plutôt international. En occident, la voiture particulière était longtemps considérée comme un mythe structurant de la société de consommation (Baudrillard, 1968; Boltanski, 1975) et plus 
spécialement apte à desservir les régions éloignées du centre et à l'habitat diffus, tandis que le transport en commun est, lui, plus particulièrement adapté aux déplacements de masse tournés vers le centre-ville (Bonnafous et al., 1993). Dans les pays en développement et émergents et vu l'absence de service de transport en commun de bonne qualité, la voiture est considérée comme le seul moyen digne des «personnes respectables». Les utilisateurs évoquent des notions liées à l'ascension sociale, comme symbole de réussite et de luxure (Gabriel Dupoy 1995). En Algérie, depuis les années 2000, les salaires ont connu une nette revalorisation dans la majorité des secteurs, notamment de l'éducation et l'enseignement supérieur, ce qui avait donné l'occasion aux familles de se doter de plusieurs voitures. Ainsi, le parc automobile a presque doublé en 15 ans, passant de 2,7 millions à plus de 5,1 millions de véhicules de 1997 à 2013, soit un taux de croissance de 88,9\% (Idres et Kaid Tilane, 2016). A Béjaia, selon le plan de circulation (2014), la voiture particulière est le modèle de déplacement motorisé le plus fréquenté. Il atteint les $10.579 \mathrm{UVP}^{6}$, soit $62.17 \%$ de l'ensemble du trafic du cordon intérieur à l'heure de pointe du matin. Le parc automobile est évalué selon le service $\mathrm{CGB}^{7}$ (2016) à 22.057 véhicules pour des fréquentations quotidiennes, ajouté à cela les véhicules issus des trafics d'échange ou de transite. Ceci accentue les problèmes de circulation telle que les congestions et saturations du réseau routier (Fig. 8).

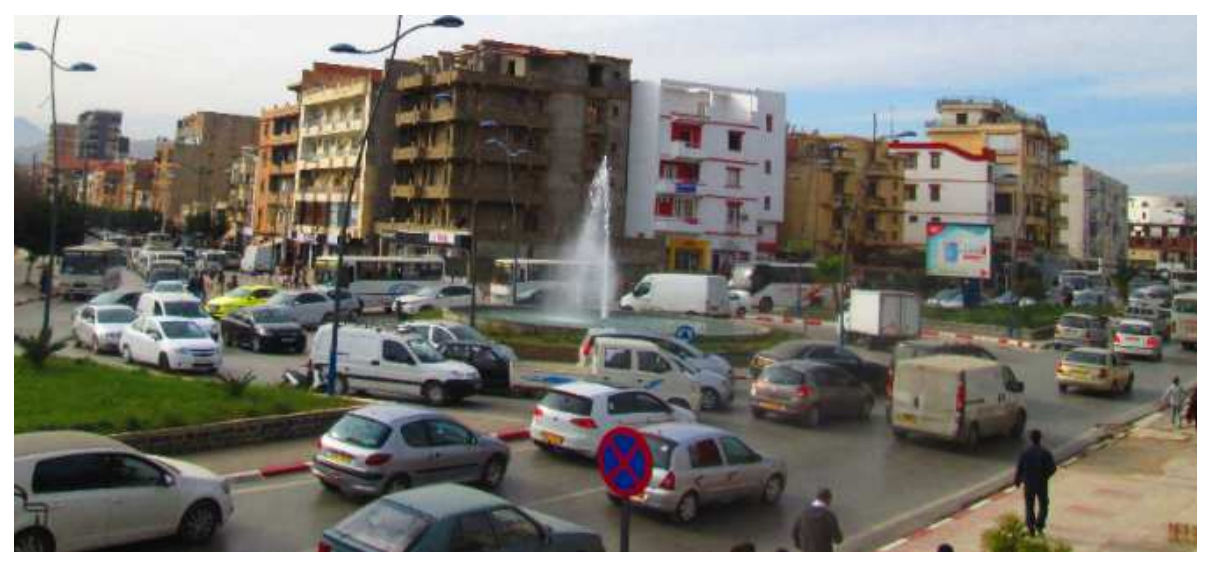

Fig. 8. Congestion au niveau du carrefour Dawadji (Source : le auteurs, 2017)

Cette situation déplorable du réseau de transport nous pousse à nous interroger sur les interventions opérées par les des autorités locales responsables afin d'y remédier?

\footnotetext{
6 UVP : Unité de Voiture Particulière

7 Service de cartes grises de la commune de Bejaia
} 


\section{Gouvernance locale en matière de gestion de la mobilité urbaine}

Le développement et l'amélioration des transports publics sont au cœur des politiques urbaines. Nombreux sont les projets de transports collectifs réalisés dans les grandes villes algériennes depuis cette dernière décennie des années 2010. Le thème des politiques de transport est une réflexion intéressante pour aborder les questions de gouvernance. La « ville durable » ne peut avoir lieu sans une prise sérieuse de la gestion des transports (Lebreton et Beaucire, 2000).

\subsection{Les politiques de gestion d'exploitation et d'entretien du réseau routier à Bejaia}

Le réseau routier est un agent déterminant pour le bon fonctionnement du système circulatoire. Le plan directeur d'aménagement et d'urbanisme intercommunal (2009) indique à ce sujet que «La circulation des flux étant au coeur de l'économie mondialisée, le sous-équipement de la région d'étude joue comme un puissant frein dans toute stratégie de développement éventuelle, rien de sérieux ne peut être fait si le réseau routier n'est pas rénové profondément. » De par sa vocation touristique, la ville de Bejaia doit être de plus en plus bien aménagée, sécurisée et accueillante en termes d'équipements touristiques, cependant les goulots d'étranglement qui se forment, le ralentissement de circulation de plus en plus ressenti en son centre ainsi que les chiffres croissants des accidents de la route dissuadent les touristes de se rendre dans la région, ce qui constitue un obstacle de taille pour son développement futur comme métropole du littoral.

Conformément à l'article 141 de la loi $\mathrm{n}^{\circ} 12-07$, le développement et l'entretien des chemins wilaya $(\mathrm{CW})$ sont à la charge des wilayas. Quant au développement et l'entretien des chemins communaux et du réseau urbain, ils sont assurés par les communes concernées conformément à l'article 149 de la loi $\mathrm{n}^{\circ} 11-10$ du code communal, plus exactement au service voirie et réseaux divers de la commune. Sachant que la commune de Bejaia dispose d'une enveloppe financière largement suffisante en matière de gestion urbaine, notamment grâce à ces activités portuaires, des sommes importantes sont allouées chaque année en faveur de projet de voirie, ce qui devra théoriquement lui permettre de se développer et corriger les disfonctionnements. Cependant, il est à remarquer que le processus d'intervention des autorités sur le terrain est complexe. Il est rendu effectif soit suite à :

1. Des réclamations citoyennes émanant des associations sociales qui signalent des problèmes au niveau de leurs quartiers,

2. Une demande hiérarchique de la part des élus. Cette demande se focalise généralement sur les axes les plus fréquentés afin d'améliorer 
la qualité de service ou pour embellir l'ambiance des principaux lieux pour les rendre plus dynamiques.

3. Diagnostiques de la part des équipes techniques de la voirie qui proposent à la hiérarchie de se pencher sur un tronçon donné vu son importance.

4. Situations d'urgence : intervenir en cas de danger tel que la fermeture d'une voie.

Donc suite à l'un des quatre cas de figure que nous venons de citer, des commissions de travaux sont tenues au siège de l'APC avec la présence des représentants politiques et techniques de plusieurs autorités concernées. La commission étudie les diverses doléances et propose des solutions, qui sont validées de commun accord et enregistrées sur un procès-verbal. Autrement dit, dans le cas où il $\mathrm{n}$ y'a pas de réclamation sur un site donné, les opérations d'amélioration risquent de ne jamais avoir lieux, notamment si le site est d'une fréquentation minime.

\subsection{Les politiques de gestion de contrôle et de prévention du trafic routier à Bejaia}

Au niveau européen, l'intégration des politiques des transports et de l'aménagement du territoire aux échelons régional, stratégique et local constitue le principal objectif des directives actuelles des documents d'orientation récents sur le transport et l'aménagement du territoire (Stead, 2003). En Algérie, cet éveil est de plus en plus ressenti dans l'élaboration des documents de gestion urbaine, qui mettent d'avantage l'accent sur la nécessité d'intégrer les facteurs environnementaux ainsi qu'une approche intégrée économique, sociale et éducative dans la politique des transports. Ainsi, les collectivités locales communales et wilayales (départementales) jouent un rôle important dans le domaine des transports urbains. Les communes sont chargées de l'organisation du réseau de transport en commun urbain ainsi que de la réglementation en matière de la circulation urbaine. Le service des transports terrestres de la Directions de Transport de Wilaya ${ }^{8}$ joue le rôle d'autorité organisatrice des transports urbains, en appliquant la réglementation relative au transport établie par le ministère des transports, et détermine le niveau de l'offre à mettre en œuvre.

La ville de Bejaia souffre de congestion et de saturation de son réseau routier, ce qui a poussé les pouvoirs publics à entreprendre la réalisation d'une série d'études relatives à l'organisation de la circulation et des transports afin d'endiguer cette congestion et de remédier au problème de la mobilité urbaine d'une manière générale. C'est dans cette visée qu'a été confiée l'étude du plan de transport et de circulation de la ville de Bejaia, en l'an 2000, au bureau

8 Les missions DTW sont fixées par le décret exécutif nº 90-381 du 24 novembre 1990 
d'étude SAETI (Société Algérienne D'études D'infrastructures). Ce dernier avait proposé plusieurs scénarii à court, moyen et long terme concernant la voirie et le transport urbain. Six ans plus tard, les collectivités communales s'étaient intéressées aux déplacements de la population Bougeotte, et ce, à travers une enquête ménages- déplacements confiée au Centre National des Technologies et de Consulting de Boumerdes (CNTC). L'objectif de cette enquête était de connaitre les pratiques de déplacements de la population afin de mesurer leurs évolutions dans le temps et d'adapter, par la suite, les politiques de transport en fonction de ces évolutions. Il s'agissait également d'interroger la population sur ses attentes en matière de transport (EMD, 2016, P.1) .

La question du réseau routier, du transport et de la circulation a aussi été largement analysée par le PDAU intercommunal. Ce dernier avait conclu que la croissance de la ville de Bejaia n'était pas harmonieuse, mais plutôt disproportionnée, ce qui nécessitait la programmation en urgence de futurs projets en faveur de la diversification des modes de transports. Le projet le plus grandiose est, sans nul doute, l'intégration d'un mode de transport doux et efficace : le tramway, appelé à relier les noyaux urbains de la plaine de Bejaia à ses deux pôles universitaires (PDAU, 2009). Vainement, les directives de 2006 n'ont pas été appliquées, ce qui a poussé les autorités locales, entre 2012 et 2014, à se pencher à nouveaux sur l'identification et la mise en exergue des dysfonctionnements du système circulatoire de la ville, et ce, via des enquêtes effectuées, cette fois-ci, par le Bureau d'Etudes des Transports filiale de l'Entreprise Métro d'Alger (BETUR). Parmi les orientations retenues pour restructurer le plan de déplacement de Bejaia, figure la mise en place de la signalisation lumineuse tricolore sur 12 carrefours névralgiques de la ville. Chose qui a été réellement effectuée en 2015 suite à la mise à jour du plan de circulation et de stationnement par le bureau d'ingénierie et d'études techniques Tichy (BETT). Quatre quartiers urbains avaient alors bénéficié de cette action : Tobal, Seghir-Aamriw, Ramla, et Ihedadden. D'autres projets ambitieux ont été aussi programmés tel que la réalisation de nouvelles trémies au centre villes, le tramway sur la plaine et le téléphérique pour les quartiers de la haute ville. Dans le même ordre d'idée, quelques recommandations ont été émises telles que la suppression des stationnements sur la chaussée et leur remplacement par des zones de stationnement payantes, qui devraient être créées à l'entrée des agglomérations pour avoir une rotation élevée, la réalisation de deux parkings sous-sol ou à étages au niveau du siège de la wilaya et de l'ancienne gare routière, ainsi que la création de parcs relais à proximité des stations de transport en commun (Aut-Sadi, 2014). Cependant suite à la restriction budgétaire annoncée par le ministère des finances, ces projets sont restés au stade théorique.

9 Enquête Ménages sur les déplacements 
Actuellement, les actions menées par la commune de Bejaia se limitent à concrétiser quelques opérations légères : dédoublement de voies et ouverture de routes secondaires, réalisation des deux trémies au niveau des carrefours, Aâmriw et Ihaddaden ainsi que l'aménagement de 19 carrefours névralgiques moyennant une enveloppe financière de 15 milliards de centimes pour l'installation des feux tricolores (Fig. 9).

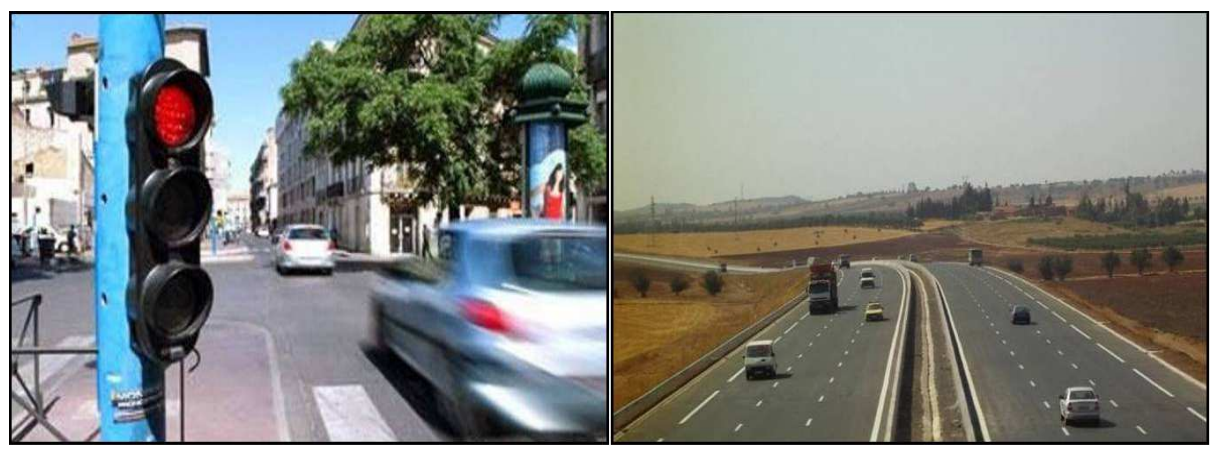

Fig. 9. Aménagement de carrefours et dédoublement des voies à Bejaia (Source : econews.com/fr/actualites/nationale/transport/l)

Le point faible de ces directives émises reste l'incapacité de trouver des mécanismes convaincants qui plaident en faveur d'une réduction du poids de l'utilisation de la voiture particulière. En effet, en l'absence de compagnes de sensibilisation en faveur de la mobilité durable et d'incitation à l'utilisation des transports collectifs, la voiture particulière reste le moyen de transport le plus utilisé par la tranche sociale aisée et même moyenne bougeotte, et ce, malgré tous les aléas environnementaux et de gestion quelle engendre. Le développement de transports publics urbains adéquats et concurrentiels à Bejaia, comme dans les autres villes algériennes d'ailleurs, ne doit pas uniquement impliquer une action résolue des pouvoirs publics, mais doit engager une approche partenariale avec les différents acteurs socio-économiques, ce qui doit constituer l'essence même de la bonne gouvernance urbaine.

\section{Conclusion}

De par sa situation stratégique et ses différents équipements socioéconomiques de grande envergure à l'échelle nationale, la ville de Bejaia connait, notamment depuis ces deux dernières décades, un étalement urbain assez important facilité et encouragé par l'augmentation du taux de possession de voitures particulières. Cet étalement, à son tour, a engendré une forte demande en matière de transport en commun. Devant l'incapacité de l'Etat de 
répondre à cette forte demande, les opérateurs privés ont pu proliférer dans l'espace urbain bougeotte provoquant ainsi dégradations sonore et visuelle de l'environnement urbain, congestion et saturation du réseau routier, initialement peu développé. Résultat : le parc véhicule, tous types confondus, de plus en plus étoffé et le circuit routier de plus en plus étouffé par d'innombrables bouchons.

De par le monde, les nouvelles instructions de planification concernant le transport plaident en faveur de l'incorporation de cette nouvelle notion de «bonne gouvernance urbaine» dans la politique urbaine, autrement dit de l'intégration de l'aménagement du territoire, et ce, afin de mieux gérer les déplacements des individus, les aléas spatio-urbains relatifs à l'étalement urbain ainsi que d'améliorer la qualité environnementale, l'accessibilité et la sécurité urbaines. Voulant intégrer cette notion de «bonne gouvernance urbaine», la ville de Bejaia a fait appel à plusieurs bureaux d'études afin de remédier aux différents problèmes de la mobilité urbaine. Les recommandations de ces études convergent vers l'adoption d'une politique volontariste, prête à innover et à modifier les comportements des citadins en matière de déplacement, d'un modèle de transport doux respectant l'environnement et le partenariat d'acteurs au service du projet. Politique et projets ambitieux qui restent, malheureusement suite à la restriction budgétaire que connait depuis ces dernières années le pays, au stade théorique. La pratique révèle plutôt de petites actions bien timides. La ville de Bejaia, via ses différents organismes étatiques et privés de gestion urbaine, doit s'adapter et trouver de nouvelles démarches de gestion stratégique prenant en compte la complexité et la diversification des régulations et des acteurs.

\section{BIBLIOGRAPHIE}

Assemblée populaire communale (APC) 2015, Étude et Constat sur le Réseau Éclairage Public de Bejaia, le bureau Études Et Réalisations tous travaux électriques et de gaz « Meziane Ridha ». Commune de Bejaia.

Auburtin, G., 1996, 'La pollution atmosphérique d'origine automobile et la santé publique', Collection Société Française de Santé, Vol. 4, Consulté le 2 janvier 2017 < http://fulltext.bdsp.ehesp.f $\mathrm{r} / \mathrm{Sfsp} /$ Rapports/pollutio.pdf $>$.

Aut saadi, S., 2014, 'Bejaia va acquérir des feux tricolores', Journal L'Eco News, no. 8, p. 5.

Azzag, E.-B., 2000, 'Projet urbain : Alger, entre le rêve et la réalité', Aménagement et Urbanisme, HABITAT et Construction, no. 8, pp. 16-24.

Baouni, T., Hadjiedj, A., 2000, 'Transport et politique urbaine dans l'agglomération d'Alger', Bulletin de l'Association de géographes français, no.77, pp. 295-304.

Baouni, T., Bakour, M., Bee, R., 2013, 'Effets de la multi-modalité à Alger sur la mobilité des usagers', Insaniyat / إنسانيات, no. 62, pp. 45-69.

Baudrillard, G., 1968, Le système des objets, Gallimard, Paris.

Beaudet, G., Wolff, P., 2003, 'La circulation, la ville et l'urbanisme : de la technicisation des transports au concept de mobilité', Vertigo no. 125, consulté le 5 décembre 2019 <https://journals.openedition.org/vertigo/11703, pp. 23-45. 
Becker, S., 2002, 'Les ficelles du métier. Comment conduire sa recherche en sciences sociales ?', Agora débats/jeunesses, no. 29, pp. 137-139.

Belouachrani, W., 2009, 'Mobilité, centralité, quelle accessibilité pour l'hyper centre d'Alger', Vies de villes, no. 12, pp. 12-19.

Benkhnnouche, L., 2012, Le transport urbain et la reconfiguration de la ville. Quelles interactions. Cas de la ville de Bejaia, Mémoire de magister, Faculté de droit et sciences économiques, Université Abderrahmane Mira de Bejaia.

Bennacer-Khaladi, F., 2011, Attractivité aux IDE : Quel rôle pour les villes en Algérie ? Cas de la ville de Béjaia, Mémoire de Magister non édité, Faculté des sciences économiques, commerciales et des sciences de gestion, Université A. Mira de Béjaia.

Blanchet, A., Gotman, A., 2007, Série "L'enquête et ses méthodes » : L'entretien (2 éd. refondue), Armand Colin Paris.

Boltanski, L., 1975, 'Les usages sociaux de l'automobile : concurrence pour l'espace et accidents'. Actes de la Recherche en sciences sociales, no. 2, pp. 25-49.

Boughedaoui, M., Chikhi, S., Driassa, N., Kerbachi, R., Joumard, R., 2009, 'Caractérisation du parc de véhicule algérien et son usage', International Symposium Proceedings Environment and Transport in differents contexts, Ghardaïa, pp. 201-208.

Berchache, R., 2011, Développement urbain et multi-modalité face aux enjeux du développement durable de l'agglomération d'Alger: Perspectives d'un challenge, Mémoire de magister en urbanisme, EPAU, Alger.

Bogdan, R., Taylor, S.-J., 1975, Introduction to qualitative research methods: a phenomenological approach to the social sciences, Wiley, New York.

Bonnafous, A., Brun, G., Faivre d'Arcier, B., 1993, La complémentarité entre la voiture particulière et les transports collectifs en zone urbaine, Rapport du groupe de travail du C.N.T. <http://temis.d ocumentation.developpement-durable.gouv.fr/docs/Temis/0032/Temis-032781/9742.pdf>.

Boutabba, H., 2001, Le lotissement légal entre la procédure officielle et la procédure parallèle. Cas de la ville de M'sila, Thèse de magistère, Institut de Gestion des Techniques Urbaines, Université de M'sila.

Boutabba, H., 2013, Spécificités spatiales et logiques sociales d'un nouveau type d'habitat domestique du Hodna oriental. Le type Diar Charpenti, Thèse de doctorat en Science, Département d'architecture, Université de Biskra.

Bureau d'etudes des transports urbains (BETUR) 2014, Plan de transport et de circulation de la ville de Bejaia, Bureau d'Études des Transports filiale de l'Entreprise Métro d'Alger.

Bureau d'etudes techniques tichy (BETT). 2015. Rapport Diagnostic Étude Détail Plan Circulation Béjaia V01 - PHASE 1 : Diagnostic de l'état actuel. Bureau d'ingénierie et d'études techniques, Tichy.

Kristian, Colletis-Wahl (Université de Savoie), Corinne Meunier (INRETE), 2003, 'Infrastructures de transport et développement économique en espace rural', Quelles méthode pour quels effet ?, 24 juin 2003, p. 06.

Cote, M., 1979, Mutations rurales en Algérie: le cas des hautes plaines de L'Est, éditions office des publications universitaires OPU, Alger.

Cote, M., 1983, L'espace algérien. Les prémices d'un aménagement, Editions office des publications universitaires OPU, Alger.

Davis, A., 1992, 'Liveable streets and perceived accident risk: quality of life issues for residents and vulnerable road users', Traffic Engineering and Control, no. 5, pp. 374-387.

Décret exécutif $n^{\circ}$ 90-381 du 24 novembre 990 relatifs à l'organisation et au fonctionnement des directions des transports des Wilayas.

De Gayffier, A., Lebreton, J., Beaucire, Francis, 2004, 'Transports publics et gouvernance urbaine', Recherches et Prévisions, no.75, pp. 112-114. 
Direction de la programmation et du suivi budgétaires (DPSB) 2016, Annuaire Statistique de la Wilaya de Bejaia, statistique de la Direction de la Programmation et du Suivi Budgétaires de la wilaya de Bejaia.

Direction des travaux publics (DTP) 2015, Statistiques sur Consistance et État du Réseau Routier, Par commune Arrêté au 31/12/2014, Direction des travaux publique.

Direction des travaux de wilaya (DTW) 2015, Les Statistique Sur Le Transport Urbain, Direction de transport de la wilaya de Bejaia.

Direction des travaux de Wilaya (DTW) 2017, Statistique Sur Le Transport Public Routier De Voyageurs, Bejaia, Direction de transport de la wilaya de Bejaia.

Duchesne, S., 2000, Pratique de l'entretien dit 'non-directif': Les méthodes au concret. Démarches, formes de l'expérience et terrains d'investigation en science politique, Presses Universitaires de France, Paris.

Dupoy, G., 1995a, Les territoires de l'automobile, Anthropos/Economica, Paris.

Dupoy, G., 1995b, La dépendance automobile : symptômes, analyses, diagnostic, traitements, Ed Economica, Paris.

Enquête ménages déplacements Bejaia EMD 2006, commune de Bejaia.

Idjraoui, O., Khaldi, Z., Boutabba, H., (dir), 2016, La place de la signalisation routière dans l'organisation du système circulatoire. Cas de la ville de Bejaia, Master académique, Institut de gestion des techniques urbaines, université de M'sila.

Idres, B., KaidTilane, N., 2016, 'La politique de transport en Algérie : Moteur de croissance économique ou de dépense ?', Cahiers du MECAS, no.13, Consulté le 2 janvier 2017, en ligne < http://dx.doi.org/>.

Kheladi, M., 2002, Analyse du système de transport dans une ville moyenne. Cas de Bejaia in Chantal Chanson-Jabeur et Saib-Musette(ed.), Transport et interurbain en Algérie, CREAD-CNRS. Alger, 137-158.

Kheladi, Bellataf M., Arhab, A., 1998, Aménagement urbain et développement socio-économique de Bejaia. Projet CNEPRU n ${ }^{\circ}$ M/0601/01/96, Laboratoire de Recherche Économie et Développement, Université de Bejaia.

Lebreton, J., Beaucire, G., 2000, Transports publics et gouvernance urbaine, l'Harmattan, Paris.

Leveque, J., Fideli, J.-P., 2017, Le patrimoine routier algérien sous surveillance, Un jumelage avec l'Union européenne en appui. RGRA, no. 948, pp. 1-125.

Loi ${ }^{\circ} 12-07$ relative a la Wilaya.

Loi n ${ }^{\circ} 11-10$ relative à la commune.

Loi 90-08 du 7 avril 1990 portant code de la commune.

Merlin, P., Choay, F., 1996, Dictionnaire de l'urbanisme et de l'aménagement, PUF, Paris.

Merzoug, S., 2017, 'Les centres urbains en Algérie : comment concilier l'attractivité et la mobilité à travers la gestion du transport urbain ? Cas de la ville de Bejaia', Recherche Transports Sécurité. Nec Plus, no. 01-02, pp. 1-16.

Merzoug, S., 2018, 'Le transport maritime face aux défis contradictoires de la mondialisation et du développement durable : quelles stratégies pour les armateurs ?' Les cahiers $d u$ CEDIMES, Vol. 12, no. 2, pp. 53-68.

Merzoug, S., Belkhiri, A., 2010, 'La problématique du financement des infrastructures de transport à la lumière de la crise financière mondiale. Cas de l'Algérie'. Séminaire international sur le transport public. Université A. Mira de Bejaia.

Messaoudene, K., Boutabba, H., 2017, Problématique routière des piétons et des personnes à mobilité réduite. Cas du Boulevard Drissi Yahia dans la ville de Bouira, Mémoire de Master académique, Institut de gestion des techniques urbaines, université de M'sila.

Michelat, G., Simon, M., 1977, Classe, religion et comportement politique, Presses de la Fondation Nationale des Sciences Politiques, Paris. 
Mira, R., 2015, Économie politique de l'industrialisation en Algérie : analyse institutionnelle en longue période, thèse de doctorat d'état en économies et finances, Université Sorbonne Paris.

Mutin, G., 1980, 'Implantations industrielles et aménagements du territoire en Algérie', Géo carrefour, Vol 55, no. 1, pp. 5-37.

Pegard, O., 2007, 'L'abribus : un procédé performatif dans la circulation de l'ordre et des images', Espaces Temps.net, Travaux, Vol. 10, no. 1, consulté le 15 mars 2007, $<$ https://www.espacestemps.net/articles/abribus/>.

Plan directeur d'aménagement et d'urbanisme (PDAU) de Bejaia 1997, SUCH, Bejaia.

Service de carte grise de la commune de Bejaia (SCG) 2016, Statistique sur le parc automobile à Bejaia.

Plan directeur d'aménagement et d'urbanisme intercommunal de Bejaia 2009, SUCH, Bejaia.

Sadoudi, S., 2013, Analyse du système des infrastructures de la circulation routière autour de la baie de Bejaia. Mémoire de Master en sciences économique, Option aménagement du territoire et développement, Faculté des sciences économiques, commerciales et des sciences de gestion, Université de Bejaia.

Sali, S., 2015, Gestion et organisation du transport collectif en milieu urbain. Cas de la ville de Bejaia, Mémoire de Master en sciences économique, Option aménagement du territoire et développement, Faculté des sciences économiques, commerciales et des sciences de gestion, Université de Bejaia.

Simiand, F., 1983, 'Méthode historique et science sociale', in Pierre Bourdieu, Jean-Claude Chamboredon, Jean-Claude Passeron (ed.), Le Métier de sociologue, Paris, Mouton, coll. « Les textes sociologiques, 125-135.

Stead, D., 2003, 'Les politiques des transports et de l'aménagement du territoire sont-elles vraiment coordonnées ?', ERES Revue internationale des sciences sociales, no. 176, pp. 371-387.

Société algérienne d'étude d'infrastructures (SAETI) 2001, étude des plans de transport et de circulation de la ville de Bejaia, Volume annexe, société algérienne d'études d'infrastructures, Bejaia. 